\title{
Comparison of the Effect of Er,Cr:YSGG Laser and Halita Mouthwash on Oral Malodor in Patients With Chronic Periodontitis: A Randomized Clinical Trial
}

\author{
Banafsheh Poormoradi $^{(\oplus)}$, Leila Gholami ${ }^{2 *}{ }^{\circledR}$, Reza Fekrazad $^{3,4}{ }^{\circledR}$, Amirarsalan Hooshyarfard $^{1}{ }^{\circledR}$, Ali Reza

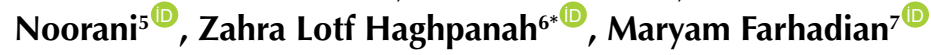 \\ 'Dental Research Center, Department of Periodontology, Dentistry Faculty, Hamadan University of Medical Sciences, \\ Hamadan, Iran \\ ${ }^{2}$ Dental Research Center, Department of Periodontology, Dentistry Faculty, Hamadan University of Medical Sciences, \\ Hamadan, Iran \\ ${ }^{3}$ Radiation Sciences Research Center, Laser Research Center in Medical Sciences, AJA University of Medical Sciences, \\ Tehran, Iran \\ ${ }^{4}$ International Network for Photo Medicine and Photo Dynamic Therapy (INPMPDT), Universal Scientific Education and \\ Research Network (USERN), Tehran, Iran \\ ${ }^{5}$ Orthodontic Department, Dentistry Faculty, Kashan University of Medical Sciences, Kashan, Iran \\ ${ }^{6}$ Dentistry Faculty, Kashan University of Medical Sciences, Kashan, Iran \\ 7Department of Biostatistics, School of Public Health and Research Center for Health Sciences, Hamadan University of \\ Medical Sciences, Hamadan, Iran
}

\section{*Correspondence to \\ Leila Gholami, \\ Assistant Professor, Dental Research Center, Department of Periodontology, Dentistry Faculty, Hamadan University of Medical Sciences, Hamadan, Iran. \\ Email:I.gholami@hotmail.com Zahra Loft Haghpanah, Dentistry Faculty, Kashan University of Medical Sciences, Kashan, Iran. Email: zahra.lotfpanah@gmail. com}

Received: October 30, 2020 Accepted: March 9, 2021 epublished: June 20, 2021

\begin{abstract}
Introduction: Periodontal disease and tongue coatings are among the major factors associated with oral malodor. The present study, comparatively evaluated the effects of the Er,Cr:YSGG laser and Halita mouthwash as adjunctive treatments to nonsurgical periodontal debridement on oral malodor reduction in chronic periodontitis patients.

Methods: Sixty patients with stage II and III chronic periodontitis and bad breath. The patients were randomly divided into two groups $(\mathrm{n}=30)$. After conventional scaling and root planing, patients in group 1 underwent Er,Cr:YSGG laser (Waterlase; Biolase, San Clemente, CA, USA) irradiation of the internal surface of the pockets ( $1.5 \mathrm{~W}, 30 \mathrm{~Hz}, 20 \% \mathrm{~A}, 40 \% \mathrm{~W}$ ) and the dorsum of the tongue $(1 \mathrm{~W}, 30 \mathrm{~Hz}, 20 \%$ A, $40 \% \mathrm{~W}$ ) immediately after SRP and on the third and seventh days. Group 2 patients were asked to use Halita mouthwash twice daily for one week. Baseline, 1 and 3-month post-treatment measurements of plaque index (PI), probing pocket depth (PPD), clinical attachment level (CAL), gingival index (GI), bleeding on probing (BOP) and organoleptic assessment of Halitosis severity were performed.

Results: Significant improvement in all parameters was noted in both groups after 1 and 3 months, compared with baseline $(P<0.05)$. The two groups had significant reductions which occurred in PPD, CAL and BOP levels and the organoleptic score in 1 and 3 months after the intervention $(P<0.05)$.

Conclusion: Er,Cr:YSGG laser irradiation and Halita mouthwash as adjuncts to non-surgical periodontal therapy are both effective in the treatment of oral malodor and improvement of periodontal parameters.

Keywords: Halitosis; Chronic periodontitis; Solid-state lasers; Mouthwashes.
\end{abstract}

\section{Introduction}

The unpleasant odor exhaled from the oral cavity is regarded as Halitosis (Bad breath). ${ }^{1-3}$ this condition is a common dilemma of many patients, influencing their social life. From an etiological perspective, $80 \%$ to $90 \%$ of the cases have been reported to be directly linked to oral and dental conditions and bacterial putrefaction of food particles in periodontal pockets or interdental areas. ${ }^{4,5}$

Volatile sulfur compounds (VSCs) produced by oral pathogenic bacteria such as Porphyromonas gingivalis, Prevotella intermedia, Treponema denticola and
Porphyromonas endodontalis are considered to be the significant contributing factor. ${ }^{5}$ Hydrogen sulfide (H2S), mainly detected on the dorsum of the tongue, is one of these compounds. Methanethiol $(\mathrm{CH} 3 \mathrm{SH})$, found in periodontal pockets. And dimethyl sulfide $(\mathrm{CH} 3 \mathrm{SCH} 3)$, which has an extraoral origin are other substances from this group. ${ }^{5,6}$ The severity of halitosis is mainly determined based on the amounts of detectable VSCs., The association of periodontal diseases and oral malodor has been confirmed in previous studies. Several studies have claimed that effective periodontal therapy can

Please cite this article as follows: Poormoradi B, Gholami L, Fekrazad R, Hooshyarfard A, Noorani AR, Lotf Haghpanah Z, et al. Comparison of the effect of er,cr:ysgg laser and halita mouthwash on oral malodor in patients with chronic periodontitis: a randomized clinical trial. $J$ Lasers Med Sci. 2021;12:e26. doi:10.34172/jlms.2021.26 
eliminate or decrease the severity of halitosis. ${ }^{8}$

Mechanical periodontal therapy has been considered a non-surgical treatment approach with a high success rate for years. Moreover, a more significant bacterial reduction and superior clinical healing have been observed in patients undergoing conventional periodontal therapy assisted with an adjunct modality.,10 Mouthwashes are commonly used as an adjunct chemical approach to control bacterial infection. Halita mouthwash is a combination of $0.05 \%$ chlorhexidine $(\mathrm{CHX})$ gluconate, $0.05 \%$ cetylpyridinium chloride (CPC) and zinc lactate. ${ }^{11}$ Studies have also shown that metal ions, such as zinc ( $\mathrm{Zn})$, inhibit the production of VSCs and subsequently decrease or eliminate halitosis. ${ }^{12}$ Also, antimicrobial agents like CHX or CPC may hinder the formation of VSCs and reduce halitosis. ${ }^{13,14}$ Furthermore, these compounds may have synergistic effects for the treatment of bacterial infections. ${ }^{11}$

Recently, some improvements were reported in the treatment of periodontal disease, and some novel modalities were introduced for this purpose. Lasers have been suggested as an adjunctive treatment of eliminating pathogenic bacteria from deep periodontal pockets. ${ }^{10,15-17}$

The Er,Cr:YSGG laser (2.78 $\mu \mathrm{m}$ wavelength) demonstrates a shallow penetration depth into tissues and a negligible thermal defect risk to deep tissues. Moreover, it has been reported that root surface treatment with this laser can result in a suitably conditioned surface which can improve the attachment of blood compounds and cells to the roots. ${ }^{18,19}$

Er,Cr:YSGG laser, combined with conventional periodontal treatment, has been reported to have positive effects in a few studies. ${ }^{18,20}$ In addition to eliminating bacteria from infected pockets, Er,Cr:YSGG laser has been applied for blood vessel coagulation and de-epithelization of periodontal pockets. ${ }^{20,21}$

Krespi et $\mathrm{al}^{22}$ have previously studied and reported the tongue as an important culprit of oral malodor, which should be considered in its treatment protocols.

In a systematic review conducted by Roncati et $\mathrm{al}^{23}$ and several other studies, positive adjunctive effects have been reported for the application of lasers for phase I periodontal debridement treatments. ${ }^{15,17,20,24,25}$

A few studies have focused on the effects of the Er,Cr:YSGG laser on periodontal therapy and oral malodor reduction. A recent study by Dereci et al has reported the effectiveness of the Er,Cr:YSGG laser in periodontal treatment and the alleviation of oral malodor. ${ }^{26}$

The current study aimed to compare the efficacy of Er,Cr:YSGG adjunctive irradiation in a periodontal therapy protocol with conventional periodontal therapy plus Halita mouthwash to treat halitosis and periodontal disease.

\section{Materials and Methods}

Sixty patients with a chief complaint of bad breath, who were referred to the Department of Periodontology, were enrolled. All patients signed informed consent forms before the study.

\section{Patient Selection}

Sixty patients consisting of 37 females and 23 males with an age range between $37.97 \pm 8.3$ years and diagnosed with Stage II (interdental CAL of 3-4 mm, probing depth $\leq 5 \mathrm{~mm}, 15-33 \%$ bone loss) and III chronic periodontitis (CAL $\geq 5 \mathrm{~mm}$ interdentally, maximum probing depth of $\geq 6 \mathrm{~mm}$, bone loss reaching the middle third of the root, a $3 \mathrm{~mm}$ or more vertical loss of bone) were included. ${ }^{22}$ Other inclusion criteria were the presence of a minimum of 15 teeth in the oral cavity and a plaque index of under $30 \%$.

The exclusion criteria consisted of (1) Antibiotic intake in the past 6 months (before treatment), (2) History of the previous or current use of anti-infective mouthwashes, (3) History of previous periodontal surgery, (4) Systemic diseases such as gastrointestinal or respiratory diseases, (5) Presence of carious teeth, (6) History of xerostomia or oral conditions such as fungal infections (based on the examination and approval of an oral disease specialist), (7) History of oral lesions, (8) Presence of tongue with deep grooves (fissured tongue) and coated tongue, and (9) Presence of any metabolic and mucocutaneous diseases.

The selected patients were divided into two groups by simple randomization (flipping a coin). Group 1: Er,Cr:YSGG irradiation of pockets and tongue was applied immediately after traditional scaling and root planing. Group 2 patients received scaling and root planing and were instructed to use Halita mouthwash afterwards for one week.

\section{Oral Hygiene Instructions}

The patients were provided with oral hygiene instructions in their first appointment. Written and verbal instructions were given to correct the method of using the toothbrush and dental floss and adhere to the precise oral hygiene measures after periodontal therapy.

\section{Treatment Modality}

In both groups, the patients received conventional periodontal treatment with an ultrasonic scaler (Wood Pecker, China) which included thorough sub- and supragingival scaling and root planning of both arches in a single session.

In group 1, the Er,Cr:YSGG laser (Waterlase, iPlus, Biolase, California, USA) was irradiated with MZ5 and MZ8 tips. The MZ5 laser tip was used with a $10^{\circ}$ angle with the root surface and applied in reciprocal movements (mesiodistal movement in an apical to coronal direction) in contact with the interior pocket wall. Only the internal surface 
of the pockets was irradiated with the laser once in each session. An output power of $1.5 \mathrm{~W}$ at a $30 \mathrm{~Hz}$ pulse rate and $20 \%$ air and $40 \%$ water with $60 \mu$ s pulse duration $(\mathrm{H}$ mode) was applied. ${ }^{26}$ In addition to periodontal pockets, the coating and bacterial burden of the tongue can be responsible for halitosis; thus, we decided to irradiate the dorsum of the tongue with the laser using the MZ8 tip with $1 \mathrm{~W}$ power, $30 \mathrm{~Hz}$ pulse rate, $20 \%$ air and $40 \%$ water with $60 \mu$ s pulse duration. The laser was held 1 $\mathrm{mm}$ away from the dorsal tongue surface and irradiated with the scanning motion. Laser irradiation was repeated three times: in the first session immediately after the conventional periodontal therapy, in the second session 72 hours after the first session, and in the third session 7 days after the first session. ${ }^{26}$

In the second group, the Halita mouthwash was prescribed after conventional periodontal therapy. 10 $\mathrm{mL}$ of the mouthwash was prescribed twice daily, and the patients were instructed to avoid eating or drinking for 60 minutes after using the mouthwash. The mouthwash was prescribed for one week only, as recommended by the manufacturer (Vi-One, Iran). ${ }^{27}$

\section{Clinical Measurements}

Probing pocket depth (PPD), clinical attachment level $(\mathrm{CAL})$, plaque index (PI), gingival index (GI) and bleeding on probing (BOP) were recorded at baseline and 1 and 3 months after the treatment. All teeth with $\mathrm{PPD} \geq 5 \mathrm{~mm}$ were irradiated in each patient. Periodontal parameters were measured at six sites around each tooth using a 1-mm scaled Williams's periodontal probe $(\mathrm{Hu}-$ Friedy, Chicago, IL, US). A single-blinded researcher recorded all measurements. The PI was measured using the criteria proposed by O'Leary, and the GI was measured according to the Loe index. ${ }^{28} \mathrm{BOP}$ was scored as positive or negative observation of bleeding evaluated 30 seconds after probing.

\section{Organoleptic Assessment}

All patients were asked to avoid drinking alcohol, smoking, or eating garlic or spicy foods a day prior to the measurements. Data were recorded at $10 \mathrm{am}$; all patients were asked to have breakfast and brush their teeth and tongue 1 hour before the measurements. These measurements were also repeated on 1 month and 3 months recall visits.

The participants were instructed to breathe only through the nose for 3 minutes, then exhale their breath slowly through their mouth. This was performed at a 10 and 30 $\mathrm{cm}$ distance from the examiner's nose. A five-degree scale, developed by Wolf et al, was used for the record of odor intensity $(0=$ no detectable odor, $1=$ barely detectable odor, 2 =slight but detectable odor, $3=$ moderate odor, $4=$ strong odor, and $5=$ extremely foul odor). ${ }^{27}$

The measurements were repeated by a single-blind expert examiner. This test was repeated in triplicates and the greatest score was considered for the patient. Menopausal women were excluded from the study, and for other women, the measurements were made after their menstrual period.

\section{Statistical Analysis}

The Shapiro-Wilk test was used for evaluating normality. The Friedman test was used for within-group comparison during the follow-up point. Also, the Mann-Whitney $\mathrm{U}$ test was applied for between-group comparisons at the re-evaluation times. For PI, PPD and CAL indexes, the repeated measures ANOVA and $t$ test were used for within- and between-group comparisons. For evaluating the GI and BOP indexes, the chi-square and Cochrane tests were also used. $P$ value $<0.05$ was considered as the level of significance.

\section{Results}

The average age of participants was $37.97( \pm 8.3)$ years with 37 females $(61.7 \%)$ and 23 males $(38.3 \%)$ and no dropouts.

No significant discrepancy existed in the periodontal parameters and the organoleptic scores between the Halita and laser groups $(P>0.05)$. All parameters significantly improved in both groups at 1 and 3 months, compared to the baseline. PPD, CAL and BOP levels and the organoleptic score at 1 and 3 months after the intervention in the laser group were also statistically reduced in comparison to the Halita group $(P<0.05$, Tables 1 and 2). However, a statistically significant difference was not found between the groups for PI at all-time points and GI at 1 month after the intervention (Tables 1 to 3 ).

\section{Discussion}

The current randomized clinical trial compared the effect of the Er,Cr:YSGG laser and Halita mouthwash as adjuncts to scaling and root planing on the reduction of oral malodor and periodontal Indexes (PI, GI, PPD, CAL, and $\mathrm{BOP}$ ) in chronic periodontitis patients.

Halitosis or oral malodor is the result of intra-oral etiologies in most cases. ${ }^{29,30}$ The intraoral factors mainly include periodontal infections and tongue coating. ${ }^{31}$ VSCs are considered as the major culprit of halitosis produced by the bacteria present on tongue biofilm, saliva and deep pockets. $^{31-33}$

Evidence shows that certain mouthwashes such as $\mathrm{CHX}$ can be used to inhibit the formation of VSCs. ${ }^{34}$ Metal ions in particular, such as $\mathrm{Zn}$, decrease or eradicate halitosis by this mechanism. ${ }^{35}$ Furthermore, antibacterial agents, particularly $\mathrm{CPC}$, have been shown to be able to inhibit VSC formation and decrease halitosis. ${ }^{36}$ Since zinc ions and antibacterial agents (CHX and CPC) exert their effects via different mechanisms of action on oral VSC inhibition, an intensified synergistic inhibitory 


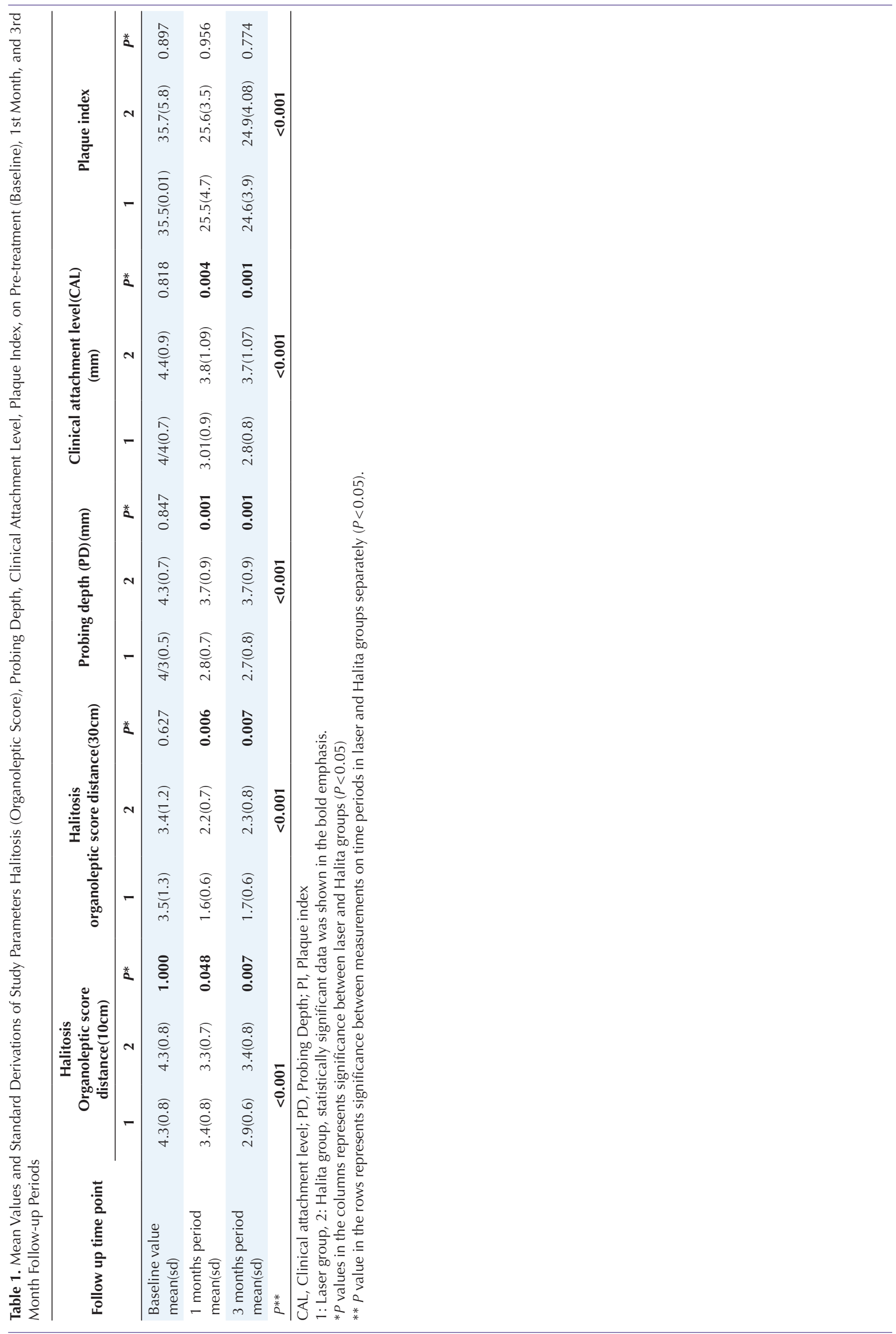


Table 2. Study Parameter for BOP (Presence or Absence of BOP)

\begin{tabular}{|c|c|c|c|c|c|c|c|c|c|}
\hline \multirow{3}{*}{ Groups } & \multicolumn{9}{|c|}{ Follow up } \\
\hline & \multicolumn{3}{|c|}{ Baseline } & \multicolumn{3}{|c|}{1 Months } & \multicolumn{3}{|c|}{3 Months } \\
\hline & Yes & No & $P^{*}$ & Yes & No & $\boldsymbol{P} *$ & yes & No & $\boldsymbol{P} *$ \\
\hline Laser & 30 & - & & 3 & 27 & & 2 & 28 & \\
\hline Halita & 30 & - & - & 11 & 19 & 0.015 & 9 & 21 & 0.02 \\
\hline$P^{* *}$ & & $<0.001$ & $<0.001$ & & & & & & \\
\hline
\end{tabular}

$* P$ values in the columns represents significance between laser and Halita groups $(P<0.05)$.

** $P$ value in the rows represents significance between measurements on time periods in laser and Halita groups separately $(P<0.05)$.

Table 3. Study parameter for Gingival Index (GI)

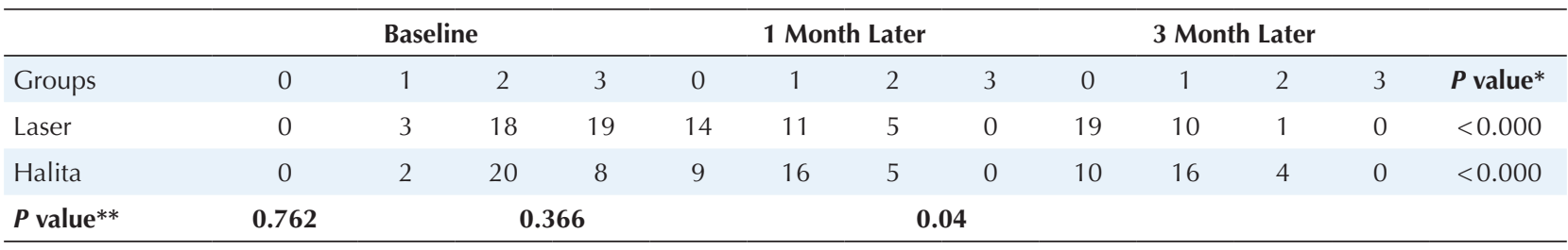

* $P$ values in the columns represents significance between laser and Halita groups $(P<0.05)$.

** $P$ value in the rows represents significance between measurements on time periods in laser and Halita groups separately $(P<0.05)$.

effect on VSC can be obtained from the combination of two or more of these agents. ${ }^{37}$ The Halita mouthwash is a combination of CHX, zinc lactate and CPC..$^{35}$

The findings of the present research indicated a statistically significant change in all periodontal parameters and organoleptic scores in the mouthwash group at all follow-up evaluation times $(P<0.05)$, which is consistent with the existing literature. ${ }^{38-40}$

Dental lasers are a new modality in dentistry; the Er,Cr:YSGG laser was introduced to the dental practice more than a decade ago and reportedly provides added periodontal regeneration benefits compared with conventional non-surgical periodontal treatment. ${ }^{16,21,41}$ Higher CAL gains have been reported for the Er,Cr:YSGG laser-irradiated group versus scaling and root planing in a previous study. ${ }^{21}$ The Er,Cr:YSGG laser applied as an adjunct to conventional periodontal treatment results in anti-bacterial effects ${ }^{42}$ and can successfully coagulate and de-epithelialize the gingival pockets. ${ }^{22}$

Dereci et $\mathrm{al}^{26}$ also observed superior anti-halitosis effects and periodontal healing in groups receiving Er,Cr:YSGG laser-assisted non-surgical periodontal therapy. The irradiation parameters in the study by these researchers were similar to those in the current research, yet the laser application method was different. In the present study, we have introduced an irradiation protocol for the treatment of halitosis, which constitutes debridement of the dorsal surface of the tongue in addition to periodontal pocket debridement. The tongue can sometimes be covered by a coating which is believed to be responsible for creating a malodor from the oral cavity. In previous studies, patients were advised to brush the dorsum of the tongue. In the present research, we decided to irradiate the dorsum of the tongue with the laser and observe its potential effect in reducing this coating and bacterial load, leading to the reduction of halitosis. ${ }^{45}$

The Er,Cr:YSGG irradiation can result in oral bacterial biofilm eradication with its antibacterial effects. Moreover, it has been shown to be more effective compared to lasers such as Nd:YAG, and this finding is in line with what has been observed in the present study. ${ }^{44,45}$ In addition, this type of laser irradiation with its minor penetration depth in oral soft tissues can be considered much safer for resurfacing the tongue.

Many types of hard lasers, namely Er,Cr:YSGG and the Er:YAG lasers, have been used for periodontal therapy. ${ }^{42,43}$ Nd:YAG lasers have deep penetration depth and may also cause a rise in temperature of the surrounding tissues. ${ }^{15,42}$ Er,Cr:YSGG lasers have considerably low thermal side effects, with extreme effectiveness and safety for periodontal therapy and oral soft tissue surgeries. ${ }^{15}$

Krespi et $\mathrm{al}^{22}$ have also recently evaluated debridement of the tongue using the Er,Cr:YSGG laser for oral malodor reduction, showing that laser tongue debridement significantly reduces malodor.

According to the results obtained by Sezen et $\mathrm{al}^{45}$ it can be deduced that the Er,Cr:YSGG laser applied along with non-surgical periodontal treatment results in the mitigation of clinical inflammation. They have pointed out that further multi-centre well-designed randomized controlled clinical trials are still required.

According to the results of the current study, both groups had a statistically significant improvement in all measured post-treatment periodontal parameters compared to the baseline $(P<0.05)$. The present study showed that PPD, BOP, CAL and organoleptic scores in the patients who underwent Er,Cr:YSGG laser treatment were significantly better compared with the Halita 
mouthwash group, but the difference between the groups was not statistically considerable for PI and GI at 1 month after the intervention. The purpose of the measurements of PI was to evaluate the oral hygiene of participants to make sure that the oral hygiene status did not have a confounding effect on the results. Our findings were consistent with those of Kelbauskiene et $\mathrm{a}^{17}$ who found a reduction in $\mathrm{PD}$ and $\mathrm{BOP}$ in patients treated with the adjunctive Er,Cr:YSGG laser versus patients treated with scaling and root planing alone. Gupta et $\mathrm{al}^{15}$ also reported significant CAL improvement for Er,Cr:YSGG-assisted periodontal treatment and open flap debridement. This finding was not consistent with the results of Gilthorpe et $\mathrm{al}^{21}$ reporting no positive effect on PD for the Er,Cr:YSGG treated group, compared with no-laser conventional treatment. However, it should be noted that Er,Cr:YSGG laser parameters in their research were different from those of the present study as Gilthorpe et al..$^{21}$ aimed to evaluate the decrease in specific periodontal pathogens after the application of the Er,Cr:YSGG laser.

\section{Conclusion}

In the present study, Er,Cr:YSGG laser irradiation and Halita mouthwash as adjuncts to conventional periodontal therapy both appeared to be safe and effective in the reduction of oral malodor and improvement of clinical periodontal parameters, with better results in the Er,Cr:YSGG laser-treated group.

\section{Funding}

Not applicable.

\section{Conflict of interests}

Each author of the present paper would like to declare that there is no conflict of interest

\section{Availability of Data and Material}

Data can be presented upon request of the editorial board of the journal.

\section{Ethical Considerations}

The study protocol was approved by the local Clinical Research Ethics Committee of Hamadan University of Medical Sciences (IR.UMSHA.REC.1397.94) and registered in the Iranian Registry of Clinical Trials (identifier: IRCT20120215009014N224).

\section{References}

1. Shimura M, Watanabe S, Iwakura M, Oshikiri Y, Kusumoto $\mathrm{M}$, Ikawa $\mathrm{K}$, et al. Correlation between measurements using a new halitosis monitor and organoleptic assessment. J Periodontol. 1997;68(12):1182-1185. doi: 10.1902/ jop.1997.68.12.1182

2. Quirynen M, Avontroodt P, Soers C, Zhao H, Pauwels M, Van Steenberghe D. Impact of tongue cleansers on microbial load and taste. J Clin Periodontol. 2004;31(7):506-510. doi: 10.1111/j.0303-6979.2004.00507.x

3. Rosenberg M, McCulloch CA. Measurement of oral malodor: current methods and future prospects.
J Periodontol. 1992;63(9):776-782. doi: 10.1902/ jop.1992.63.9.776.

4. Amir E, Shimonov R, Rosenberg M. Halitosis in children. J Pediatr. 1999;134(3):338-343. doi: 10.1016/s00223476(99)70460-9

5. Liu P-F, Zhu W-H, Huang C-M. Vaccines and photodynamic therapies for oral microbial-related diseases. Curr Drug Metab. 2009;10(1):90-94.

6. Tangerman A, Winkel E. The portable gas chromatograph OralChroma $^{\text {mox }}$ : a method of choice to detect oral and extra-oral halitosis. J Breath Res. 2008;2(1):017010. doi: 10.1088/1752-7155/2/1/017010.

7. Rosenberg M, Kulkarni G, Bosy A, McCulloch C. Reproducibility and sensitivity of oral malodor measurements with a portable sulphide monitor. J Dent Res. 1991;70(11):1436-1340. doi: 10.1177/00220345910700110801.

8. Pham TAV, Ueno M, Zaitsu T, Takehara S, Shinada K, Lam $\mathrm{P}$, et al. Clinical trial of oral malodor treatment in patients with periodontal diseases. J Periodontal Res. 2011;46(6):722-729. 10.1111/j.1600-0765.2011.01395.x

9. Quirynen M, Zhao H, Soers C, Dekeyser C, Pauwels M, Coucke $\mathrm{W}$, et al. The impact of periodontal therapy and the adjunctive effect of antiseptics on breath odorrelated outcome variables: a doubleblind randomized study. J Periodontol. 2005;76(5):705-712. doi: 10.1902/ jop.2005.76.5.705.

10. Saglam M, Kantarci A, Dundar N, Hakki SS. Clinical and biochemical effects of diode laser as an adjunct to nonsurgical treatment of chronic periodontitis: a randomized, controlled clinical trial. Lasers Med Sci. 2014;29(1):37-46. doi: 10.1007/s10103-012-1230-0.

11. Asadollahi A, Taghavi A, Eslami H, Aghazadeh M, Zeinalzadeh E, Kafil HS. Comparing Antimicrobial Effect of CO2 Laser with Halita in Oral Infection Control. Biomed pharmacol J. 2016;9(2):787-791. doi: 10.13005/bpj/1004.

12. Motahhari P. A Comparison of antimicrobial effect of the mouthwash containing chlorhexidine, Cetylpyridinium chloride, and zinc lactate (Halita) and chlorhexidine against Pseudomonas aeruginosa and staphylococcus aureus: in vitro study. Sch Bull. 2:547-553.doi: 10.21276/ sb.2016.2.10.1

13. Mann-Whitney U. Effect of Halita mouthwash on oral halitosis treatment: A randomized triple-blind clinical trial. J Dent Res Dent Clin Dent Prospects. 2019;13(1):31-35.

14. Jamali Z. Comparison of efficacy of Halita mouthrine with chlorhexidine mouthrine in reducing oral malodor [dissertation]. Tabriz, Tabriz University of Medical Sciences; 2018.

15. Gupta M, Lamba AK, Verma M, Faraz F, Tandon S, Chawla $\mathrm{K}$, et al. Comparison of periodontal open flap debridement versus closed debridement with Er,Cr:YSGG laser. Aust Dent J. 2013;58(1):41-49. doi: 10.1111/adj.12021.

16. Kelbauskiene S, Maciulskiene V. A pilot study of Er, Cr: YSGG laser therapy used as an adjunct to scaling and root planing in patients with early and moderate periodontitis. Stomatologija. 2007;9(1):21-26.

17. Kelbauskiene S, Baseviciene N, Goharkhay K, Moritz A, Machiulskiene V. One-year clinical results of Er, Cr: YSGG laser application in addition to scaling and root planing in patients with early to moderate periodontitis. Lasers Med 
Sci. 2011;26(4):445-452. doi: 10.1007/s10103-010-0799-4.

18. de Oliveira GJPL, Cominotte MA, Beraldo TPP, Sampaio JEC, Marcantonio RAC. A microscopic analysis of the effects of root surface scaling with different power parameters of Er, Cr: YSGG laser. Microsc Res Tech.. 2015;78(6):529-535. doi: 10.1002/jemt.22505.

19. Iwai K, Shi Y-W, Endo M, Ito K, Matsuura Y, Miyagi M, et al. Penetration of high-intensity Er: YAG laser light emitted by IR hollow optical fibers with sealing caps in water. Appl Opt. 2004;43(12):2568-2571. doi: 10.1364/AO.43.002568.

20. Gutknecht N, Van Betteray C, Ozturan S, Vanweersch L, Franzen R. Laser supported reduction of specific microorganisms in the periodontal pocket with the aid of an Er,Cr:YSGG laser: a pilot study. Sci World J. 2015;2015:450258. doi:10.1155/2015/450258

21. Gilthorpe MS, Zamzuri AT, Griffiths GS, Maddick IH, Eaton KA, Johnson NW. Unification of the "burst" and "linear" theories of periodontal disease progression: a multilevel manifestation of the same phenomenon. J Dent Res. 2003;82(3):200-205. doi:10.1177/154405910308200310.

22. Krespi YP, Kizhner V, Wilson KA, Sivriver A, Low S, Khosravi Y, Stoodley P. Laser tongue debridement for oral malodor-A novel approach to halitosis. Am J Otolaryngol. 2020 Mar 13:102458.

23. Roncati M, Gariffo A. Systematic review of the adjunctive use of diode and Nd: YAG lasers for nonsurgical periodontal instrumentation. Photomed Laser Surg. 2014;32(4):186197. doi: 10.1089/pho.2013.3695.

24. Dyer B, Sung EC. Minimally invasive periodontal treatment using the Er,Cr: YSGG laser. A 2-year retrospective preliminary clinical study. Open Dent J. 2012;6:74-78. doi: $10.2174 / 1874210601206010074$.

25. dos Santos Romero S, Schalch TO, do Vale KL, Ando ES, Mayer MPA, Feniar JPG, et al. Evaluation of halitosis in adult patients after treatment with photodynamic therapy associated with periodontal treatment: Protocol for a randomized, controlled, single-blinded trial with 3-month follow up. Medicine. 2019;98(39). doi: 10.1097/ MD.0000000000016976.

26. Dereci O, Hatipoglu M, Sindel A, Tozoglu S, Ustun K. The efficacy of Er,Cr:YSGG laser supported periodontal therapy on the reduction of peridodontal disease related oral malodor: a randomized clinical study. Head Face Med. 2016;12(1):20. doi: 10.1186/s13005-016-0116-y.

27. Wolf H, Rateitschak-Pluss E, Rateitschak K. Color atlas of dental medicine: periodontology. Georg Thieme Verlag Stuttgart; 2005.

28. Mishra V, Shettar L, Bajaj M, Math AS. Comparison of a commercially available herbal and $0.2 \%$ chlorhexidine mouthrinse for prevention of oral malodor: A clinical trial. J Int Soc Prev Community Dent. 2016;6(Suppl 1):S6-s11. doi: 10.4103/2231-0762.181160.

29. Scully C, Greenman J. Halitosis (breath odor). Periodontol. 2008;48:66-75. doi: 10.1111/j.1600-0757.2008.00266.x.

30. Miyazaki H, Sakao S, Katoh Y, Takehara T. Correlation between volatile sulphur compounds and certain oral health measurements in the general population. J Periodontol. 1995;66(8):679-684. doi: 10.1902/jop.1995.66.8.679.

31. Tonzetich J, Richter VJ. Evaluation of volatile odoriferous components of Saliva. Arch Oral Biol. 1964;9:39-46. doi: 10.1016/0003-9969(64)90042-1.

32. Tonzetich J, Eigen E, King WJ, Weiss S. Volatility as a factor in the inability of certain amines and indole to increase the odour of saliva. Arch Oral Biol. 1967;12(10):1167-1175. doi: 10.1016/0003-9969(67)90065-9.

33. Tonzetich J, Kestenbaum RC. Odour production by human salivary fractions and plaque. Arch Oral Biol. 1969;14(7):815-827. doi: 10.1016/0003-9969(69)90172-1.

34. Eley BM. Antibacterial agents in the control of supragingival plaque--a review. Br Dent J. 1999;186(6):286-296. doi: 10.1038/sj.bdj.4800090.

35. Roldan S, Herrera D, Santa-Cruz I, O'Connor A, Gonzalez I, Sanz M. Comparative effects of different chlorhexidine mouth-rinse formulations on volatile sulphur compounds and salivary bacterial counts. J Clin Periodontol. 2004;31(12):1128-1134. doi: 10.1111/j.1600051X.2004.00621.x.

36. Rosenberg M, Gelernter I, Barki M, Bar-Ness R. Daylong reduction of oral malodor by a two-phase oil:water mouthrinse as compared to chlorhexidine and placebo rinses. J Periodontol. 1992;63(1):39-43. doi: 10.1902/ jop.1992.63.1.39.

37. Carvalho M, Tabchoury C, Cury J, Toledo S, NogueiraFilho G. Impact of mouthrinses on morning bad breath in healthy subjects. J Clin Periodontol. 2004;31(2):85-90. doi: 10.1111/j.0303-6979.2004.00452.x.

38. Quirynen M, Zhao H, van Steenberghe D. Review of the treatment strategies for oral malodour. Clin Oral Investig. 2002;6(1):1-10. doi: 10.1007/s00784-002-0152-9.

39. Quirynen M, Avontroodt P, Soers C, Zhao H, Pauwels M, Coucke W, et al. The efficacy of amine fluoride/stannous fluoride in the suppression of morning breath odour. $J$ Clin Periodontol. 2002;29(10):944-54. doi: 10.1034/j.1600051x.2002.291010.x.

40. Söder B, Johansson B, Söder P. The relation between foetor ex ore, oral hygiene and periodontal disease. Swed Dent $J$. 2002;24(3):73-82.

41. Pavone C, Perussi LR, de Oliveira GJ, Scardueli CR, Cirelli JA, Cerri PS, et al. Effect of Er,Cr:YSGG laser application in the treatment of experimental periodontitis. Lasers Med Sci. 2015;30(3):993-999. doi: 10.1007/s10103-014-1526-3.

42. Kara C, Demir T, Orbak R, Tezel A. Effect of Nd: YAG laser irradiation on the treatment of oral malodour associated with chronic periodontitis. Int Dent J. 2008;58(3):151-158. doi: 10.1111/j.1875-595X.2008.tb00191.x.

43. Papapanou PN, Sanz M, Buduneli N, Dietrich T, Feres M, Fine DH, Flemmig TF, Garcia R, Giannobile WV, Graziani F, Greenwell H. Periodontitis: Consensus report of workgroup 2 of the 2017 World Workshop on the Classification of Periodontal and PeriImplant Diseases and Conditions.J Periodontol. 2018 Jun; 89:S173-82. Doi: 10.1002/JPER.17-0721

44. Sezen D, Hatipoğlu M, Üstün K. Evaluation of the clinical and biochemical efficacy of erbium, chromium: ytriumscandium-gallium-garnet (ER, CR: YSGG) laser treatment in periodontitis. Lasers Med Sci. 2020;35(7):1567-1575. doi: 10.1007/s10103-020-02990-8. 